\title{
Evaluation of Internal Resistance in Asphalt Concretes
}

\author{
Yousef Zandi ${ }^{1), *}$, and Muhammet Vefa Akpinar ${ }^{2)}$
}

(Received July 30, 2012, Accepted September 27, 2012)

\begin{abstract}
Composites are somewhat more difficult to model than an isotropic material such as iron or steel due to the fact that each layer may have different orthotropic material properties. In finite element literature the asphalt mixes are represented by using rectangular meshes, not the actual picture of their cross-sections. Asphalt aggregate size and distribution in the asphalt concrete sample, aggregate shape, and fractured surface effects are ignored. In this research, the actual image of the sample including all these effects were directly considered in the finite element. The samples, were cut into cross-sections and were scanned. The image-processing toolbox of Labview was utilized in obtaining the rectangular gray images of the scanned images. In the rectangular sample the aggregates were white and the asphalt binders were black. The grayscale images were converted by LABVIEW into the format required by ANSYS as an input file, with the same dimensions. The nodes at the bottom of the model were constrained in both $\mathrm{x}$ and $\mathrm{y}$ directions. Left and right edges were symmetry and top was free. Certain amount of pressure was applied along the top surface to simulate the tire pressure.
\end{abstract}

Keywords: asphalt concrete, aggregate gradation, image analysis, FEM.

\section{Introduction}

A major concern in asphalt pavement roads is excessive permanent deformation (rutting) resulting from heavy truck loads. Rutting appears as longitudinal depressions in the wheel paths and increases with increasing numbers of load applications. Safety concerns such as steering problems and decrease in bearing capacity on asphalt pavements are the results of rutting. The asphalt pavement layer must exhibit high resilient moduli and show low permanent deformation in order to reduce and rutting in the asphalt pavement.

Rutting (permanent deformation) in an asphalt-concrete layer is caused by a combination of densification (volume change) and shear deformation. Shear deformations caused primarily by large shear stresses in the upper portions of the asphalt-aggregate layer(s) are dominant.

Granular materials have a major function in the structural capacity of a highway pavement. Because approximately $85 \%$ of the total volume of asphalt concrete mixtures consist of aggregates, the performance of the asphalt concrete is greatly affected by the properties of the aggregate blend

\footnotetext{
${ }^{1)}$ Department of Civil Engineering, Tabriz Branch, Islamic Azad University, Tabriz, Iran.

*Corresponding Author; E-mail: zandi_engineer@yahoo.com

${ }^{2)}$ Department of Civil Engineering, Karadeniz Teknik Universitesi, Trabzon, Turkey.

Copyright $($ The Author(s) 2012. This article is published with open access at Springerlink.com
}

(Ahlrich 1996; Ahlrich 1995; Zakaria and Less 1996). They provide a foundation that supports asphalt concrete layers and helps to support the pavement. One of the most important properties of an aggregate blend is its size gradation, which defines the percentages of different particle sizes that are present in the blend. Gradation affects almost all the asphalt concrete properties, including durability, stability, stiffness, permeability, workability, fatigue resistance, frictional resistance and moisture damage.

The primary objective of 2002 AASHTO (American Association of State Highway and Transportation Officials) was to advance the state-of-the-practice from empirical to mechanistic related design procedure. AASHTO Joint Task Force on Pavements (JTFOP) to initiated an study objective of developing mechanistic pavement analysis suitable for use in future versions of the AASHTO guide.

Rutting associated test techniques for asphalt pavement materials are empirical. It is important that a mechanistic procedure be developed which will reasonably predict the permanent deformation under loading by heavy traffic.

Finite element (FE) analyses provide significant basis for the development of mechanistic analysis. Available FE programs are powerful tools for studying stress-strain analysis in pavement structure. Pavement rutting cannot be estimated with sufficient accuracy and reliability using current mechanistic procedures which are based on either (1) linear viscoelastic models or (2) layer-strain algorithms. However, FE techniques are now available that are well adapted to the analysis of permanent deformation in pavement structures. They can effectively handle complex constitutive relationships as well as the transverse distribution of traffic. 


\section{Objective}

In order to determine the rutting of the asphalt pavement layer for a given mix type, permanent deformation parameters of the mix can be developed from laboratory testing. These parameters can then be used to predict the permanent deformation of the material taken in the field and from the image and FEM analyis is the optimum use of unbound granular materials as a structural layer in pavements can be determined. In this research, the actual image of the sample were directly considered in the FE. In the FE literature the asphalt mixes are represented by using rectangular meshes, not the actual picture of their cross-sections.

\section{Materials and Methods}

\subsection{Sample Preparation}

The preparation of the samples were followed according to superpave protocols. Fifteen centimeter diameter samples were prepared by a superpave gyratory compactor. Aggregate gradations used in this study were coarse.

\subsection{Image Analysis}

The samples were cut into horizontal cross-sections. These cross-sections were scanned at resolutions from 400 to $600 \%$ of actual size. Certain details were lost below and above these ranges. The image-processing toolbox of LABVIEW was utilized in obtaining the rectangular gray images (the smallest dimension is at least four times the maximum aggregate size of the scanned images). In the rectangular sample the aggregates were white and the asphalt binders black.

The grayscale image of Fig. 1b was converted by LABVIEW into Fig. 2a which was the format required by ANSYS as an input file, with the same dimensions.

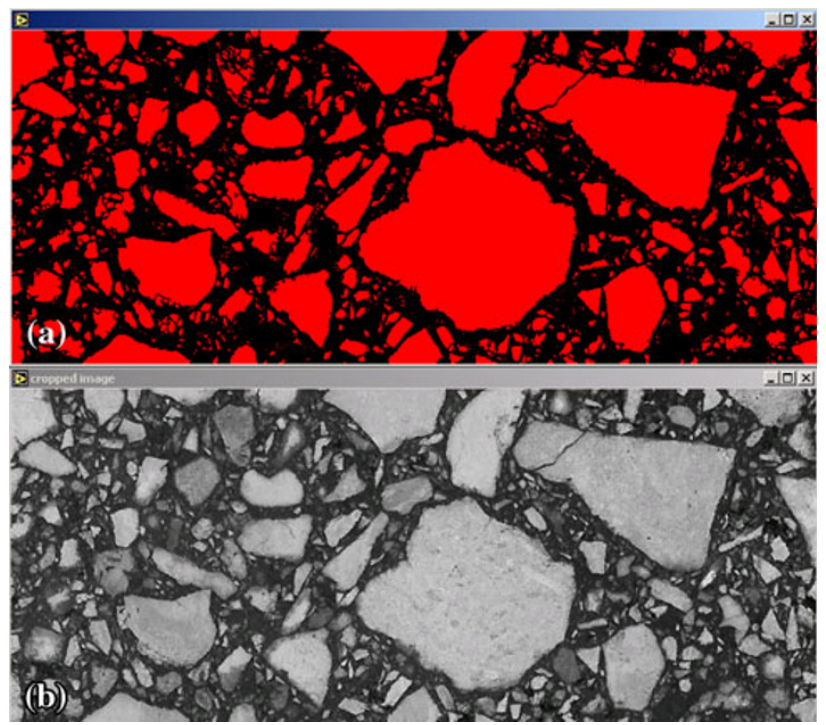

Fig. 1 a Sample image taken from the cross-section image to be converted to LABVIEW. b Cross-section image of coarse graded sample at $400 \%$ scale.

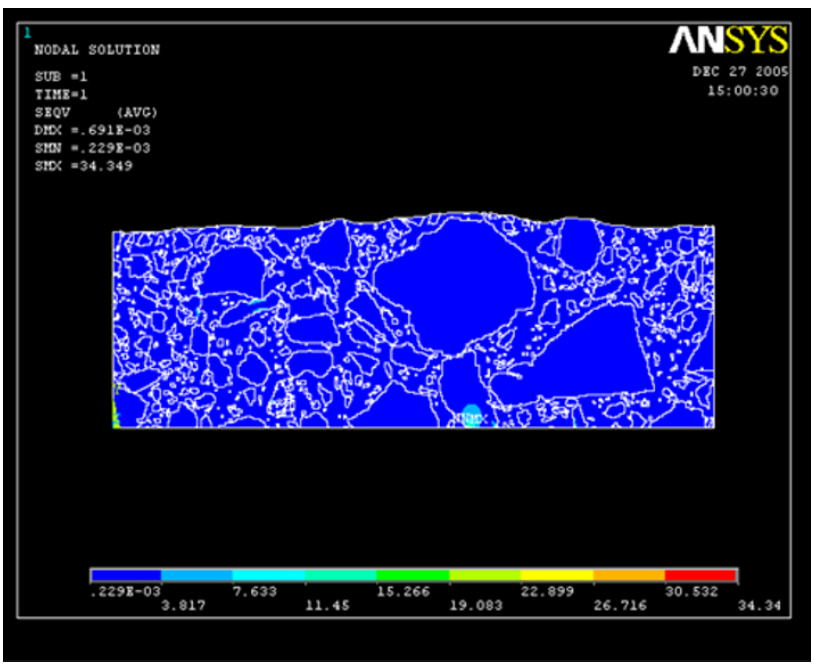

Fig. 2 Vertical displacements and after loading.

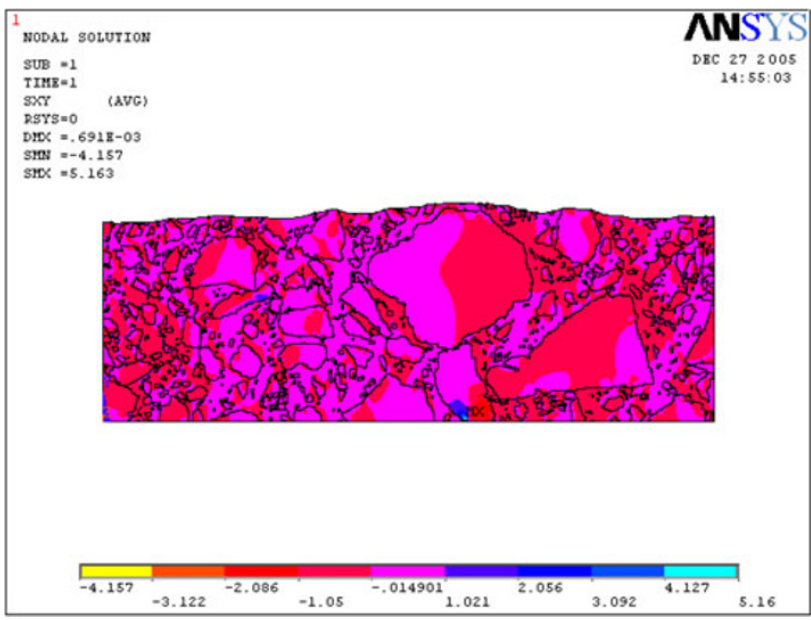

Fig. 3 Shear stress distribution.

\subsection{FE Analysis}

In this research the general purpose FE method ANSYS (1998) was selected as a numerical tool which was useful when calculating the stress and strains for the transferred image from the cut asphalt pavement core. A 2-D FEM structure similar to Fig. 3 was constructed. The following sections are described in the order that they were used in the FEM. Table 1 shows the typical materials properties assigned.

\subsubsection{Element}

The FEM model consist of 62,012 $(148 \times 419)$ elements. The asphalt pavement layer was represented by PLANE82 element. PLANE82 is a higher order version of the twodimensional, four-node element (PLANE42). The 8-node element is defined by eight nodes having two degrees of freedom at each node: translations in the nodal $\mathrm{x}$ and $\mathrm{y}$ directions. The element may be used as a plane element or as an axisymmetric element. The element has plasticity, creep, swelling, stress stiffening, large deflection, and large strain capabilities. 
Table 1 Material properties that were assigned in the ANSYS FEM model.

\begin{tabular}{c|c|c}
\hline & Modulus of elasticity (MPa) & Poisson's ratio \\
\hline \hline Asphalt & 15 & 0.4 \\
\hline Aggregate & 20,000 & 0.2 \\
\hline
\end{tabular}

\subsubsection{Meshing}

Mesh size and configuration is and important part of FE modeling, precise mesh refinement being necessary in regions of high stress intensity. However, as the mesh was made finer, the number of elements increased resulting in increased memory and computational time requirements. The software was limited to 150,000 nodes. Thus, some modifications (smaller dimension) were required in the modeling process.

\subsubsection{Boundary Conditions}

The boundary conditions of the model were as follows. The nodes at the bottom of the model were constrained in both $x$ and $y$ directions. The bottom line was constrained in the $\mathrm{X}, \mathrm{Y}$ directions. The outer edges were allowed only in the vertical direction. Material properties (elastic modulus, Poisson's ratio) of mix were entered. Homogenous linear load pressure of $700 \mathrm{kPa}$ representing the tire was positioned on the top surface.

\subsubsection{Observation of Sample Failure}

It was observed that at the end of the load permanent deformation test, the deformation of the image sample visually demonstrated the rut depth. Figure 2 shows the mixes at the end of permanent deformation testing at $2.1 \mathrm{~kg} /$ $\mathrm{cm}^{2}$ stress level. The shear stresses due to the vertical pressure are shown in Fig. 3. It is clear from the figure that the shear stress varies through the sample representing the actual field conditions.

It can be seen that the sample made of the virgin mix is seriously damaged, the sample made of the $1.5 \%$ modified mix is also deformed, but nowhere near the extent shown for

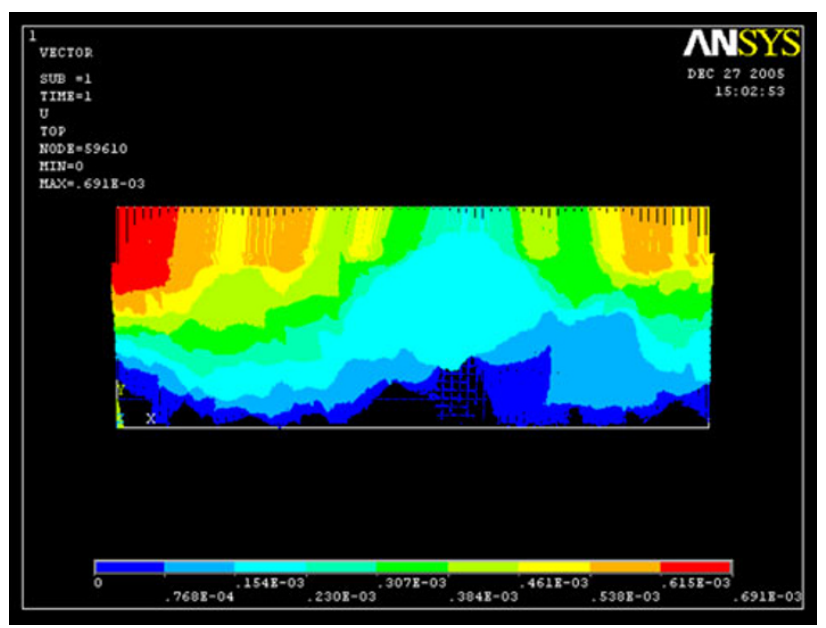

Fig. 4 Vectoral analyses. the virgin mix. Computed vertical deformation of $0.5 \mathrm{~mm}$ was obtained. The found value is lower than expected.

The computed ruth depth value is underestimated since the FEM stress are computed in two dimensional instead of three dimensional. Higher rut depths are expected when the model were to be constructed in three dimensional model environment. Due to memory limitations three dimensional model was not constructed.

From Fig. 4 it can be said that the sample under loading not only moves in the vertical direction but also in the horizontal direction which is the definition of the rutting. The aggregate materials are forced to move in the horizontal and vertical shear forces.

Surface irregularities after loading is due to the fact that the elastic modulus and poisson ratios through the sample was not the same. In regular pavement FEM programs it is assumed to be homogenous. In reality it is not due to the varying aggregate gradation in the asphalt concrete. Aggregate gradation is very important for the skeleton which carries most of the trafficload especially in the summer where asphalt binder stiffness is too low.

The measured ruth depths and the shear strength from triaxial testing, for the same samples, the simulated ANSYS FE will be compared in order to verify accuracy of model to complete the mechanistic empirical study suggested by AASHTO.

\section{Conclusion}

In this study a mechanistic study using a FE tool was used to reasonably predict the rut depth under loading. Actual picture of cut asphalt concrete core sample cross-sections were transferred to FEM mesh environment. Asphalt aggregate size and distribution in the asphalt concrete sample, aggregate shape, and fractured surface effects were included in the FEM analysis. Further studies are being conducted to verify the found rut depth so that future study will be full mechanistic-empirical analysis. One can have the best aggregate gradation for rutting, but the aggregates in the sample could be distributed in such a way that a strong aggregate skeleton may not be obtained. Improved images can anticipate the arrangement of the skeleton.

\section{Open Access}

This article is distributed under the terms of the Creative Commons Attribution License which permits any use, distribution, and reproduction in any medium, provided the original author(s) and the source are credited. 


\section{References}

Ahlrich, R. C. (1995). Influence of aggregate gradation and particle shape/texture on permanent deformation of hot mix asphalt pavements. Ph.D dissertation, Auburn University, Auburn, AL.

Ahlrich, R. C. (1996). Influence of aggregate properties on performance of heavy-duty hot-mix asphalt pavements. Transportation Research Record, 1547, 7-14.
ANSYS. (1998). ANSYS elements reference, ANSYS 5.5 (10th ed.). Canonsburg: ANSYS Inc.

Zakaria, M., \& Less, G. (1996). Rutting characteristics of unbound aggregate layers. Construction and Building Materials, 10(3), 185-189. 